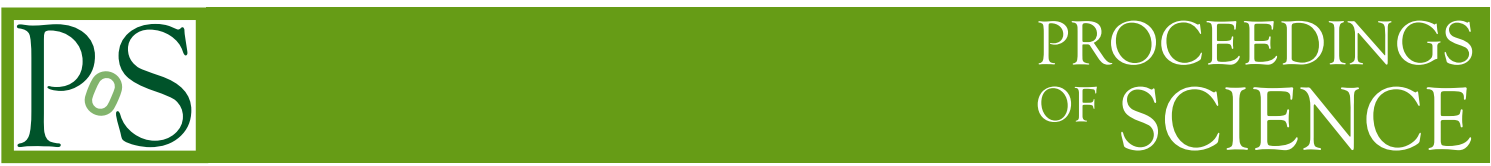

\title{
Cosmic Ray Physics with the IceCube Observatory
}

\author{
The IceCube Collaboration \\ http://icecube.wisc.edu/collaboration/authors/icrc15_icecube \\ E-mail: timo.karg@desy.de
}

\begin{abstract}
The IceCube Neutrino Observatory at the geographic South Pole comprises the cubic-kilometer deep-ice detector as well as a square-kilometer particle detector at the surface, IceTop. This unique combination allows measuring multiple components of cosmic-ray induced air showers in the $\mathrm{PeV}$ to $\mathrm{EeV}$ energy range: IceTop samples the electromagnetic component at ground level and enables studies of $\mathrm{GeV}$ muons in the periphery of the air shower; the deep-ice detector is sensitive to $\mathrm{TeV}$ muons in the shower core and in addition has collected a high-statistics sample of atmospheric muons from cosmic rays in the tens to hundreds $\mathrm{TeV}$ energy range. I will review recent cosmic-ray results obtained from IceCube data, including the cosmic ray energy spectrum and mass composition with three years of IceCube data, studies of the anisotropy at different energies, and investigations on the muon content of air showers.
\end{abstract}

Corresponding author: T. Karg*

DESY, Platanenallee 6, 15738 Zeuthen, Germany

The 34th International Cosmic Ray Conference,

30 July- 6 August, 2015

The Hague, The Netherlands

${ }^{*}$ Speaker. 


\section{Introduction}

The IceCube Observatory, installed at the geographic South Pole, not only allows the measurement of atmospheric and astrophysical neutrinos, but also serves as an excellent instrument to study cosmic rays. IceCube comprises a detector component in the deep-ice, consisting of 86 vertical strings installed in boreholes of $2500 \mathrm{~m}$ depth. The bottom $1000 \mathrm{~m}$ of each string are instrumented with 60 digital optical modules (DOMs) each that detect the Cherenkov light emitted by charged particles traversing the detector [1]. In addition, the IceTop surface array serves as an air shower detector. IceTop comprises 81 stations at an altitude of $2835 \mathrm{~m}$ a.s.l. $\left(X \approx 680 \mathrm{~g} \mathrm{~cm}^{-2}\right)$ that are installed at the top of the strings of the deep-ice detector with a typical horizontal spacing of $125 \mathrm{~m}$. Each station has two ice-Cherenkov tanks, separated by $10 \mathrm{~m}$, with a diameter of $1.8 \mathrm{~m}$ which are filled with $0.9 \mathrm{~m}$ of clear ice. The tank is instrumented with two DOMs operating at different gain to cover a dynamic range from about $1 / 6$ VEM (vertical equivalent muon) to 1140 VEM [2]. The IceCube Observatory has been completed in December 2010, but already with partial detector configurations physics analyses were possible during the construction phase.

\section{Observation Modes}

In this section the different possibilities for detecting various components of air showers with the IceCube Observatory are discussed. Recent results employing these observation modes are presented in Sec. 3.

The IceTop surface array is triggered when six tanks in three stations register a signal in coincidence. The signal in the triggering tanks is typically dominated by the electromagnetic component of air showers. On every trigger generated by IceTop or the deep-ice detector, the signals from all tanks and the deep-ice detector are stored. IceTop has a small, central in-fill array with a threshold of about $100 \mathrm{TeV}$ primary cosmic-ray energy, and the regular spaced array has a threshold of $300 \mathrm{TeV}$. It records air showers from primary cosmic rays of energies up to about $2 \mathrm{EeV}$ above which the rate becomes too low for analysis. The direction of events passing standard selection cuts can be reconstructed with an uncertainty of about $0.2^{\circ}$ at $30 \mathrm{PeV}$; the energy resolution for protons at this energy is 0.05 in $\log _{10}(E / \mathrm{GeV})$ [2]. Using only data from the IceTop array, the all-particle energy spectrum is derived, the anisotropy of $\mathrm{PeV}$ cosmic rays is studied, and searches for point-like sources of neutrons are performed.

The low trigger threshold of the individual IceTop tanks of 1/6 VEM allows detailed studies of the periphery of extensive air showers. At large distances from the shower core the signal in the tanks will be dominated by single muons. The coincidence between two tanks in a station, required for the array trigger, typically will not be fulfilled, but the data of these single tanks will be read out and stored on an air shower trigger. This allows measuring the GeV muon content of an air shower and supplies a handle to study cosmic-ray composition and hadronic interaction models. In addition, muon-poor showers can be selected as candidate events in searches for a diffuse flux, or point-like sources of $\mathrm{PeV}$ photons.

The deep-ice detector component is sensitive to high energy muons produced early in the air shower development. Vertical muons with a surface energy larger than about $1 \mathrm{TeV}$ can trigger the deep-ice detector and this energy threshold increases with increasing zenith angle, i.e. growing 

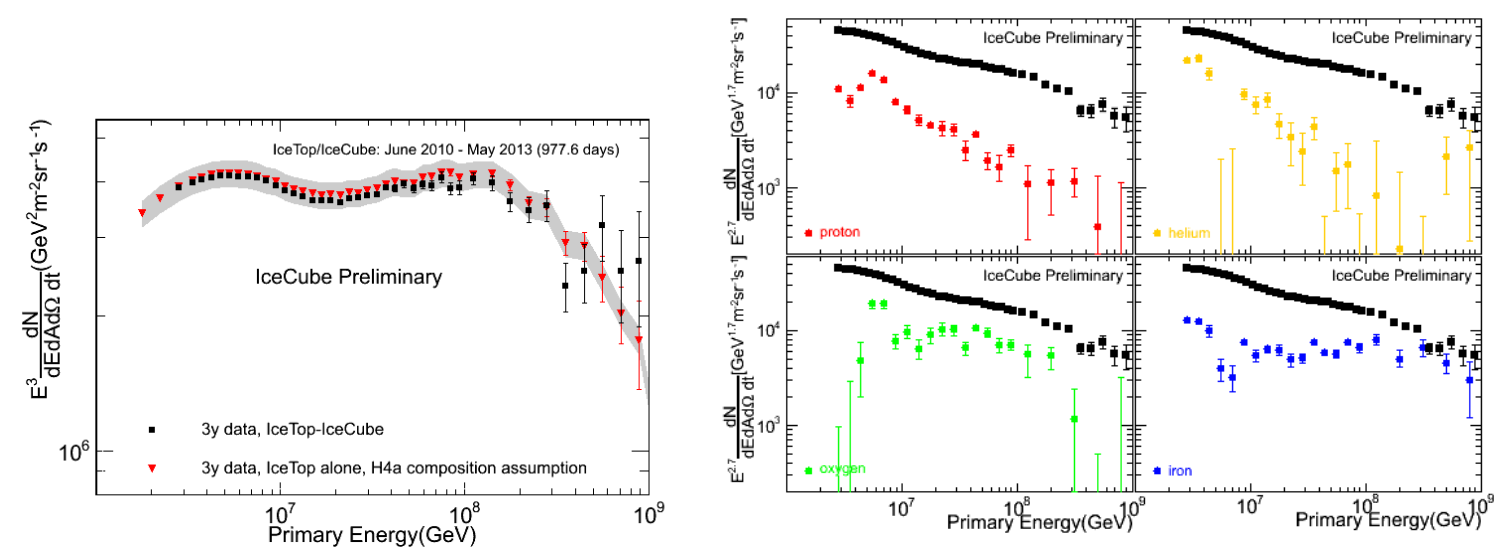

Figure 1: Left: All-particle energy spectrum based on three years of IceCube data. The red markers show the IceTop-alone analysis; the grey band represents the systematic uncertainty due to the unknown composition. The black markers show the result of the spectrum and composition analysis using coincident events in IceTop and the deep-ice detector [3]. Right: Energy spectra for four individual mass groups measured from coincident events [3].

overburden of ice. Due to the large detector volume, an unprecedented statistics of about $10^{11}$ atmospheric muon events per year is collected. These atmospheric muons enable studies of the cosmic-ray anisotropy above $1 \mathrm{TeV}$. At higher cosmic-ray energies, the central muon core of the air shower can penetrate down to the deep-ice detector. The amount of light measured in the deep-ice detector allows to estimate the number of muons in the bundle, which in turn is related to the mass number $A$ of the primary cosmic particle. Another, independent handle on cosmic-ray composition and hadronic interactions models.

Finally, the information from IceTop and the deep-ice detector can be combined on an eventby-event basis, leading to more precise measurements, but limiting the available zenith range to $\theta<30^{\circ}$ since the shower axis must pass through both detector components. Combining the cosmicray primary energy measured in IceTop with the size of the $\mathrm{TeV}$ muon bundle yields energy spectra for different mass groups of primary cosmic rays. Well-reconstructed showers in IceTop with a shower axis intersecting the deep-ice detector, but with no measurable $\mathrm{TeV}$ muons are good candidates for PeV photons. And in reverse, muons in the deep-ice detector whose track direction passes through IceTop, but which have no corresponding air shower in IceTop, are good candidates for astrophysical neutrinos interacting in the ice between the two detector components.

\section{Results}

\subsection{All-Particle Spectrum and Composition of Primary Cosmic Rays}

The energy of primary cosmic rays is inferred from the reconstructed shower size at a distance of $125 \mathrm{~m}$ from the shower axis, $S_{125}$. At this distance, the shower size depends least on the mass of the primary particle. The shower size is mapped to primary cosmic-ray energy using CORSIKA [4] simulations with SYBILL 2.1 [5] and FLUKA [6] as high-energy and low-energy hadronic interaction models respectively. The efficiency of IceTop as a function of primary energy is determined with a detailed detector simulation, using Geant4 [7] to model the tank response and the effect of 

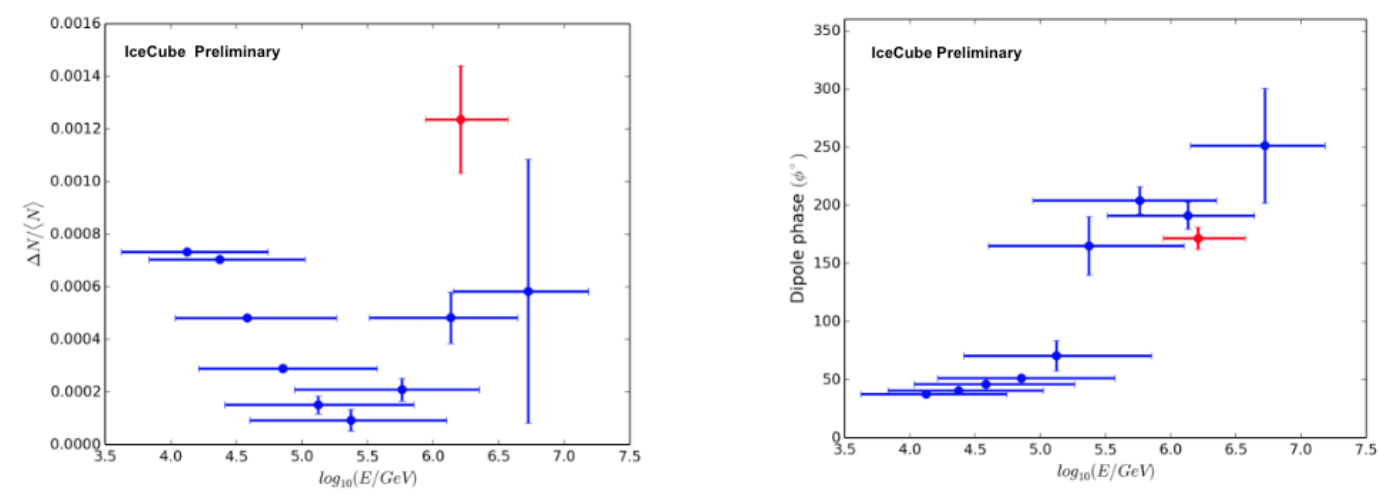

Figure 2: Amplitude (left) and phase (right) of the dipole anisotropy as function of primary cosmic ray energy. The data points mark the median energy of each subsample; the horizontal error bars show the $68 \%$ containment interval. Blue data points are obtained from the deep-ice detector; red data points are from IceTop [16].

snow on top of the tanks. An energy spectrum using one year of data of the partially completed IceTop detector with 73 tanks was previously published [8]. At this conference, an update based on three years of data (June 2010 to May 2013) was presented [3]. Figure 1 (left) shows the all-particle spectrum for the IceTop-alone analysis (red markers).

The analysis of air showers in IceTop where in addition the $\mathrm{TeV}$ muon core passes through the deep-ice detector allows the measurement of the primary mass. A first analysis based on one month of data with the 40-string, 40-station partial detector configuration has been published for primary energies up to $30 \mathrm{PeV}$ [9]. The analysis technique has been refined and applied to three years of data. It uses an artificial neural network to map the shower size $S_{125}$, the cosine of the zenith angle $\cos \theta$, the muon bundle energy-loss in the ice $\mathrm{d} E_{\mu} / \mathrm{d} X$ at $X=1500 \mathrm{~m}$ slant depth, and the number of high-energy stochastic energy losses in the ice under two selections to the primary energy and a proxy of the primary mass. Histograms of the mass proxy are generated for small intervals in reconstructed energy and are fitted with template histograms derived from Monte Carlo simulations of four different mass groups (proton, helium, oxygen, iron), using a binned likelihood fit. This way the fraction of the different mass groups for each bin of reconstructed energy is determined [3]. Figure 1 (left) shows the resulting all-particle spectrum (black markers). It is in good agreement with the IceTop-alone result. In Fig. 1 (right) the individual spectra for the four different mass groups are shown. The heavy components, represented by oxygen and iron, maintain a hard spectrum up to higher energies than proton and helium. The systematic uncertainties of the measurement are discussed in [3].

\subsection{Anisotropy}

Cosmic-ray induced air showers above about $10 \mathrm{TeV}$ can produce muons that can be measured and reconstructed with the deep-ice detector. Between May 2009 and May 2014, IceCube recorded more than $2.5 \cdot 10^{11}$ muon events that allow detailed studies of the anisotropy as function of angular scale [10, 11, 12, 13], time [14], energy, and possibly primary mass. In addition, IceTop can be used to study anisotropies in the PeV energy range [15]. 


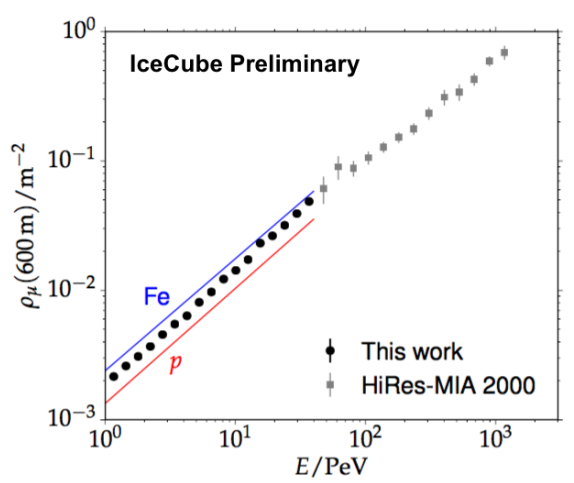

Figure 3: Measured muon density at $600 \mathrm{~m}$ from the shower axis in near vertical showers $\left(\theta \approx 13^{\circ}\right)$ [17]. Simulations with SYBILL 2.1 and FLUKA are shown for reference. The data are in good agreement with the HiRes-MIA measurement [18], which was performed at a different atmospheric depth of $X \approx 860 \mathrm{~g} \mathrm{~cm}^{-2}$.

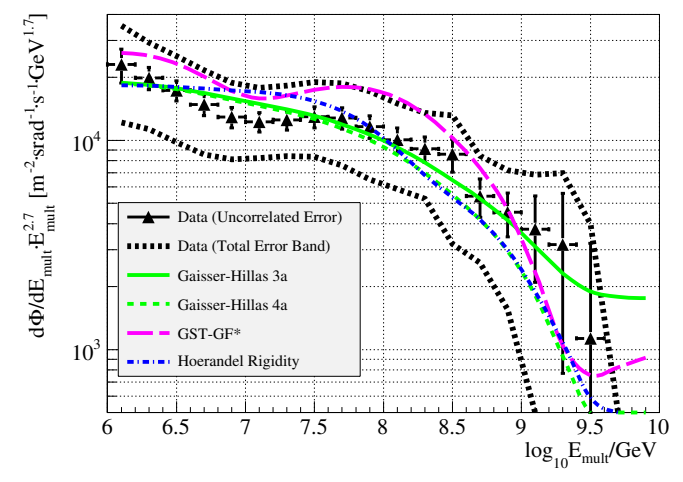

Figure 4: Muon multiplicity spectrum (cf. main text for definition) measured with one year of IceCube data. The error bars include systematic uncertainties that are uncorrelated between bins and statistical uncertainties; the error band represents correlated uncertainties (overall scaling effects) [19]. Different cosmic-ray models are shown for comparison $[20,21,22]$.

In Fig. 2 the amplitude (left) and phase (right) of the dipole component of the anisotropy is shown. It can be seen that the energy resolution is poor since only a small fraction of the primary cosmic ray energy is transported by the muons and fluctuations of the muon energy loss is the detector are large. Still, the data can be separated in nine independent energy bins with increasing median energy. One data point (in red) obtained with IceTop at $1.7 \mathrm{PeV}$ median energy is also displayed. The figure shows that a rapid shift of phase by almost $180^{\circ}$ occurs at an energy between $130 \mathrm{TeV}$ and $240 \mathrm{TeV}$. The dipole amplitude decreases with energy up to these energies, and increases again at higher energies. While the phase of the IceTop data agrees well with that found in the deep-ice data at similar energies, the amplitude of the anisotropy is larger in IceTop. This could indicate a difference in the energy distribution and the chemical composition of deep-ice and IceTop events and is currently under study [16].

\subsection{Air Shower Physics}

Low energy $(\mathrm{GeV})$ muons in the periphery of air showers give an additional handle on primary mass and the systematic uncertainties introduced by hadronic interaction models. The low trigger threshold of the individual tanks of about 1/6 VEM allows determining muon densities on a statistical basis at large distances from the shower axis where the tank signal is not longer dominated by electromagnetic particles. For a sample of showers with equal reconstructed energy and zenith angle, tanks have been binned in distance to the shower axis and measured charge. At large distances (depending on the primary energy) two populations of tanks can be identified: tanks with a charge of approx. 1 VEM that have registered a muon and tanks with $\ll 1$ VEM measuring electromagnetic particles. Slices at constant distance are fitted with a model for electromagnetic and muonic signals, taking into account multiple muons and the angular response of the tank to muons [17]. Figure 3 shows the measured muon density at $600 \mathrm{~m}$ from the shower axis. The measurement 
is independent of air shower simulations. Simulations with SYBILL 2.1 [5] and FLUKA [6] are given for reference and the data are well bracketed by proton and iron simulations.

In the future, the measured muon lateral distribution function will be added to the IceTop reconstruction and the electromagnetic and muonic component of the shower can be reconstructed separately on an event-by-event basis. This is expected to improve IceCube's sensitivity to the mass of the primary cosmic particle considerably. First results of the improved reconstruction are presented in [23].

High energy (TeV) muons can be produced with large transverse momentum $\left(p_{T}\right)$. These muons will separate from the air shower core during flight and can be measured as distinct double tracks (core muons + high- $p_{T}$ muon) in the deep-ice detector [24]. The typical transverse momentum to produce laterally separated muons in IceCube is about $2 \mathrm{GeV}$, where parton interactions can be described in the context of perturbative quantum chromodynamics (pQCD). The analysis and simulation techniques for high- $p_{T}$ muons have been refined and the sensitivity to cosmic ray composition has been studied [25].

High energy muon bundles can be measured with the deep-ice detector over a much wider zenith angle range, and thus with much higher statistics, than what can be used for the coincident composition analysis (cf. Sec. 3.1). In this mode, a direct measure for the primary cosmic ray energy can not be obtained, but tests of cosmic ray models are possible. The relevant observables are the energy loss per length of the muon bundle in the ice and large stochastic energy losses. The latter allow to separate high energy single muons from muon bundles. Using air shower simulations, these observables are related to the muon multiplicity $E_{\text {mult }}:=E_{\text {prim }} \cdot(A / 56)^{\frac{1-\alpha}{\alpha}}$, where $E_{\text {prim }}$ is the primary cosmic ray energy, $A$ is the mass number, and $\alpha=0.79$ is an empirical parameter derived from simulation [19]. Figure 4 shows the muon multiplicity spectrum measured with $1.2 \cdot 10^{7}$ muon bundle events from the 79 string configuration of IceCube. The data are compared to cosmic ray composition models from [20,21, 22], which have been translated to $E_{\text {mult }}$ using the equation given above.

\subsection{Searches for Neutral Particles}

With the IceCube Observatory searches for high energy neutrons, photons, and neutrinos have been performed.

Neutron-induced air showers cannot be distinguished from proton-induced showers. Since neutrons carry no electric charge they are not deflected in magnetic fields and a neutron source within the decay length of the particles would show up as an excess in the event distribution in celestial coordinates. With typical cosmic ray energies between 10 and $100 \mathrm{PeV}$, IceTop is sensitive to neutron sources within a distance up to about $1 \mathrm{kpc}$. Two searches have been performed. A binned all sky search using all cosmic-ray events in the zenith range $\theta<37^{\circ}$ with energies higher than $10 \mathrm{PeV}$ revealed no statistically significant clustering. Figure 5 shows the upper limit on the neutron flux at $90 \%$ confidence level as a function of declination derived with four years of IceTop data [26]. A targeted search with an energy threshold of $100 \mathrm{PeV}$ for neutrons from close-by millisecond pulsars, $\gamma$-ray pulsars, and high mass x-ray binaries showed no statistically significant correlations [26].

PeV photons are searched for by looking for muon-poor showers. A search has been performed with the 40 string configuration in the declination region $\delta<-60^{\circ}$ [28]. No correlation of 


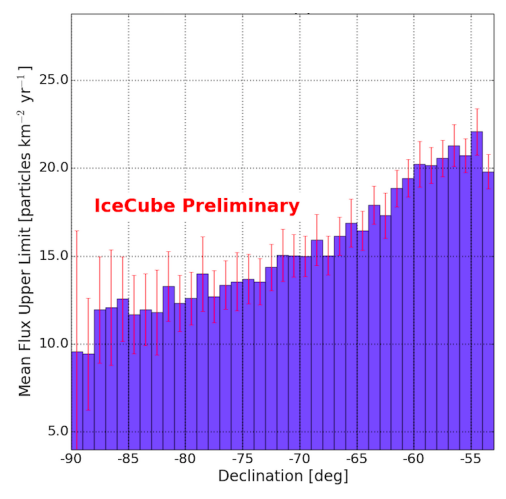

Figure 5: Upper limit (90\% C.L.) on the flux of neutrons from point-like sources as a function of declination. The error bars indicate the statistical uncertainty [26].

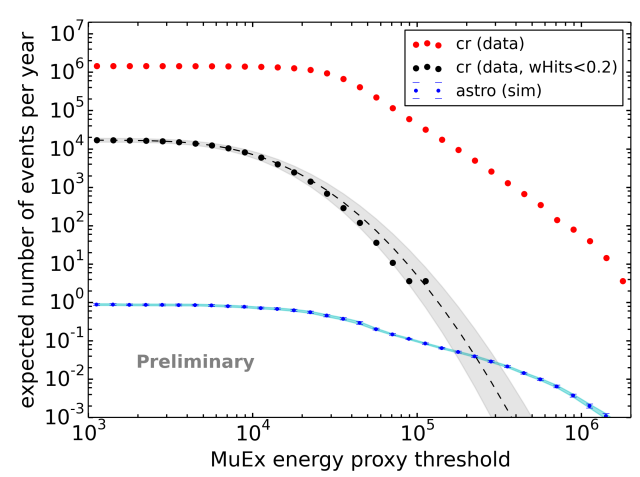

Figure 6: Atmospheric muon bundle event rates as function of the energy measured by the deep-ice detector before and after applying IceTop as active veto. The expected flux of astrophysical neutrinos is shown for reference [27].

photon candidates with the Galactic plane was found and an upper limit on the photon fraction of $1.2 \cdot 10^{-3}$ was set in the energy range from 1.2 to $6 \mathrm{PeV}$. Further, no clustering of photon candidate events was observed in a search for point-like sources in the complete field of view. A similar analysis with the full IceCube detector is in preparation.

Neutrino searches in the Southern hemisphere suffer from atmospheric muons as dominant background. This has been mitigated in previous searches by using part of the instrumentation of the deep-ice detector as an active veto, thus reducing its effective mass. If IceTop can be used as an air shower veto, the whole ice volume below IceTop and above the deep-ice detector can be utilized as additional interaction target for astrophysical neutrinos. A study of the veto efficiency for IceTop has been performed [27]. Figure 6 shows the possible reduction of the cosmic ray background by using IceTop information. While the expected astrophysical neutrino rate after applying the veto is as low as 0.1 events per year due to the small solid angle for coincident events, the analysis demonstrates the capability and requirements of a surface veto array for the IceCube-Gen2 project.

\section{Future Plans}

A new collaboration, IceCube-Gen2, has been founded in April 2015 to pursue the design and construction of the IceCube-Gen 2 facility at the South Pole. It is envisioned to comprise a low-energy array, PINGU, for precision neutrino measurements, a $\sim 10 \mathrm{~km}^{3}$ high-energy deep-ice array to study the astrophysical neutrino flux [29] and a large area surface array consisting of a $10 \mathrm{~km}^{2}$ cosmic-ray detector and a $\sim 100 \mathrm{~km}^{2}$ air shower veto of less sophisticated detection units. The cosmic-ray detector together with the high-energy deep-ice array, due to the largely increased range of zenith angles for coincident events, is expected to boost the coincident data rate by a factor of 50 and enables lateral and production depth muon measurements for every event [30], promising the most precise studies of the transition region from Galactic to extra-galactic cosmic rays. 


\section{References}

[1] F. Halzen and S. Klein, Rev. Sci. Instrum. 81 (2010) 081101.

[2] IceCube Collaboration, R. Abbasi et al., Nucl. Instrum. Meth. A 700 (2013) 188-220.

[3] IceCube Collaboration. POS(ICRC2015)334 these proceedings.

[4] D. Heck, J. Knapp, J. N. Capdevielle, et al., CORSIKA: A Monte Carlo code to simulate extensive air showers, Tech. Rep. FZKA 6019, Forschungszentrum Karlsruhe, 1998.

[5] E.-J. Ahn, R. Engel, T. Gaisser, et al., Phys. Rev. D 80 (2009) 094003.

[6] A. Ferrari, P. Sala, A. Fassó, et al., FLUKA: a multi-particle transport code, 2005. CERN-2005-10 (2005), INFN/TC_05/11, SLAC-R-773.

[7] S. Agostinelli et al., Nucl. Instrum. Meth. A 506 (2003) 250-303.

[8] IceCube Collaboration, M. Aartsen et al., Phys. Rev. D 88 (2013) 042004.

[9] IceCube Collaboration, R. Abbasi et al., Astropart. Phys. 42 (2013) 15-32.

[10] IceCube Collaboration, R. Abbasi et al., Astrophys. J. 718 (2010) L194-L198.

[11] IceCube Collaboration, R. Abbasi et al., Astrophys. J. 746 (2012) 33.

[12] IceCube Collaboration, R. Abbasi et al., Astrophys. J. 740 (2011) 16.

[13] IceCube Collaboration, M. Aartsen et al., Astropart. Phys. 66 (2015) 39-52.

[14] IceCube Collaboration, Study of the time-dependence of the cosmic-ray anisotropy with AMANDA and IceCube, in Proceedings of the 33rd International Cosmic Ray Conference, (Rio de Janeiro, Brazil), July, 2013. arXiv:1309.7006.

[15] IceCube Collaboration, M. Aartsen et al., Astrophys. J. 765 (2013) 55.

[16] IceCube Collaboration. POS(ICRC2015)274 these proceedings.

[17] IceCube Collaboration. POS(ICRC2015)267 these proceedings.

[18] T. Abu-Zayyad et al., Phys. Rev. Lett. 84 (2000) 4276-4279.

[19] IceCube Collaboration, M. Aartsen et al., submitted to Astropart. Phys. (2015) [arXiv:1506.07981].

[20] T. Gaisser, Astropart. Phys. 35 (2012) 801-806.

[21] T. Gaisser, T. Stanev, and S. Tilav, Frontiers of Physics 8 (2013) 748-758.

[22] J. Hörandel, Astropart. Phys. 19 (2003) 193-220.

[23] IceCube Collaboration. POS(ICRC2015)338 these proceedings.

[24] IceCube Collaboration, R. Abbasi et al., Phys. Rev. D 87 (2013) 012005.

[25] IceCube Collaboration. POS(ICRC2015)256 these proceedings.

[26] IceCube Collaboration. POS(ICRC2015)250 these proceedings.

[27] IceCube Collaboration. POS(ICRC2015)1086 these proceedings.

[28] IceCube Collaboration, M. Aartsen et al., Phys. Rev. D 87 (2013) 062002.

[29] IceCube-Gen2 Collaboration. POS(ICRC2015)1146 these proceedings.

[30] IceCube-Gen2 Collaboration. POS(ICRC2015)694 these proceedings. 